\title{
Measurement of dijet cross sections in ep interactions with a leading neutron at HERA
}

\section{Journal Article}

\section{Author(s):}

H1 Collaboration; Aktas, A.; Baumgartner, S.; Berger, N.; Erdmann, Wolfram; Grab, Christoph; List, Benno; Mangano, Salvatore; Meer, David; Schöning, André; Weber, R.; et al.

\section{Publication date:}

2005-06

\section{Permanent link:}

https://doi.org/10.3929/ethz-b-000031958

\section{Rights / license:}

Creative Commons Attribution 4.0 International

\section{Originally published in:}

The European Physical Journal C 41(3), https://doi.org/10.1140/epjc/s2005-02227-8 


\title{
Measurement of dijet cross sections in $e p$ interactions with a leading neutron at HERA
}

\author{
The H1 Collaboration
}

A. Aktas ${ }^{10}$, V. Andreev ${ }^{26}$, T. Anthonis ${ }^{4}$, S. Aplin ${ }^{10}$, A. Asmone ${ }^{34}$, A. Babaev ${ }^{25}$, S. Backovic ${ }^{31}$, J. Bähr $^{39}$ A. Baghdasaryan ${ }^{38}$, P. Baranov ${ }^{26}$, E. Barrelet ${ }^{30}$, W. Bartel ${ }^{10}$, S. Baudrand ${ }^{28}$, S. Baumgartner ${ }^{40}$, J. Becker ${ }^{41}$, M. Beckingham ${ }^{10}$, O. Behnke ${ }^{13}$, O. Behrendt ${ }^{7}$, A. Belousov ${ }^{26}$, Ch. Berger ${ }^{1}$, N. Berger ${ }^{40}$, J.C. Bizot ${ }^{28}$, M.O. Boenig ${ }^{7}$, V. Boudry ${ }^{29}$, J. Bracinik ${ }^{27}$, G. Brandt ${ }^{13}$, V. Brisson ${ }^{28}$, D.P. Brown ${ }^{10}$, D. Bruncko ${ }^{16}$, F.W. Büsser ${ }^{11}$, A. Bunyatyan ${ }^{12,38}$, G. Buschhorn ${ }^{27}$, L. Bystritskaya ${ }^{25}$, A.J. Campbell ${ }^{10}$, S. Caron ${ }^{1}$, F. Cassol-Brunner ${ }^{22}$, K. Cerny ${ }^{33}$, V. Chekelian ${ }^{27}$, J.G. Contreras ${ }^{23}$, J.A. Coughlan ${ }^{5}$, B.E. Cox ${ }^{21}$, G. Cozzika ${ }^{9}$, J. Cvach ${ }^{32}$, J.B. Dainton ${ }^{18}$, W.D. Dau ${ }^{15}$, K. $\operatorname{Daum}^{37,43}$, B. Delcourt ${ }^{28}$, R. Demirchyan ${ }^{38}$, A. DeRoeck ${ }^{10,45}$, K. Desch ${ }^{11}$, E.A. DeWolf ${ }^{4}$, C. Diaconu ${ }^{22}$, V. Dodonov ${ }^{12}$, A. Dubak ${ }^{31}$, G. Eckerlin ${ }^{10}$, V. Efremenko ${ }^{25}$, S. Egli ${ }^{36}$, R. Eichler ${ }^{36}$, F. Eisele ${ }^{13}$, M. Ellerbrock ${ }^{13}$, E. Elsen ${ }^{10}$, W. Erdmann ${ }^{40}$, S. Essenov ${ }^{25}$, P.J.W. Faulkner ${ }^{3}$, L. Favart ${ }^{4}$, A. Fedotov ${ }^{25}$, R. Felst ${ }^{10}$, J. Ferencei ${ }^{10}$, L. Finke ${ }^{11}$, M. Fleischer ${ }^{10}$, P. Fleischmann ${ }^{10}$, Y.H. Fleming ${ }^{10}$, G. Flucke ${ }^{10}$, A. Fomenko ${ }^{26}$, I. Foresti ${ }^{41}$, J. Formánek ${ }^{33}$, G. Franke ${ }^{10}$, G. Frising ${ }^{1}$, T. Frisson ${ }^{29}$, E. Gabathuler ${ }^{18}$, E. Garutti ${ }^{10}$, J. Gayler ${ }^{10}$, R. Gerhards ${ }^{10}{ }^{\dagger}$, C. Gerlich $^{13}$, S. Ghazaryan ${ }^{38}$, S. Ginzburgskaya ${ }^{25}$, A. Glazov ${ }^{10}$, I. Glushkov ${ }^{39}$, L. Goerlich ${ }^{6}$, M. Goettlich ${ }^{10}$, N. Gogitidze $^{26}$, S. Gorbounov ${ }^{39}$, C. Goyon ${ }^{22}$, C. Grab ${ }^{40}$, T. Greenshaw ${ }^{18}$, M. Gregori ${ }^{19}$, G. Grindhammer ${ }^{27}$, C. Gwilliam $^{21}$, D. Haidt ${ }^{10}$, L. Hajduk ${ }^{6}$, J. Haller ${ }^{13}$, M. Hansson ${ }^{20}$, G. Heinzelmann ${ }^{11}$, R.C.W. Henderson ${ }^{17}$, H. Henschel ${ }^{39}$, O. Henshaw ${ }^{3}$, G. Herrera ${ }^{24}$, I. Herynek ${ }^{32}$, R.-D. Heuer ${ }^{11}$, M. Hildebrandt ${ }^{36}$, K.H. Hiller ${ }^{39}$, D. Hoffmann ${ }^{22}$, R. Horisberger ${ }^{36}$, A. Hovhannisyan ${ }^{38}$, M. Ibbotson ${ }^{21}$, M. Ismail ${ }^{21}$, M. Jacquet ${ }^{28}$, L. Janauschek ${ }^{27}$, X. Janssen ${ }^{10}$, V. Jemanov ${ }^{11}$, L. Jönsson ${ }^{20}$, D.P. Johnson ${ }^{4}$, H. Jung ${ }^{20,10}$, M. Kapichine ${ }^{8}$, M. Karlsson ${ }^{20}$, J. Katzy ${ }^{10}$, N. Keller ${ }^{41}$, I.R. Kenyon ${ }^{3}$, C. Kiesling ${ }^{27}$, M. Klein ${ }^{39}$, C. Kleinwort ${ }^{10}$, T. Klimkovich ${ }^{10}$, T. Kluge ${ }^{10}$, G. Knies ${ }^{10}$, A. Knutsson ${ }^{20}$, V. Korbel ${ }^{10}$, P. Kostka ${ }^{39}$, R. Koutouev ${ }^{12, \dagger}, K_{\text {Krastev }}^{35}$, J. Kretzschmar ${ }^{39}$, A. Kropivnitskaya ${ }^{25}$, K. Krüger ${ }^{14}$, J. Kückens ${ }^{10}$, M.P.J. Landon ${ }^{19}$, W. Lange ${ }^{39}$, T. Laštovička ${ }^{39,33}$, P. Laycock ${ }^{18}$, A. Lebedev ${ }^{26}$, B. Leißner ${ }^{1}$, V. Lendermann ${ }^{14}$, S. Levonian ${ }^{10}$, L. Lindfeld ${ }^{41}$, K. Lipka ${ }^{39}$, B. List ${ }^{40}$, E. Lobodzinska ${ }^{39,6}$, N. Loktionova ${ }^{26}$, R. Lopez-Fernandez ${ }^{10}$, V. Lubimov ${ }^{25}$, A.-I. Lucaci-Timoce ${ }^{10}$, H. Lueders ${ }^{11}$, D. Lüke ${ }^{7,10}$, T. Lux ${ }^{11}$, L. Lytkin ${ }^{12}$, A. Makankine ${ }^{8}$, N. Malden ${ }^{21}$, E. Malinovski ${ }^{26}$, S. Mangano ${ }^{40}$, P. Marage ${ }^{4}$, R. Marshall ${ }^{21}$, M. Martisikova ${ }^{10}$, H.U. Martyn ${ }^{1}$, S.J. Maxfield ${ }^{18}$, D. Meer ${ }^{40}$, A. Mehta ${ }^{18}$, K. Meier ${ }^{14}$, A.B. Meyer ${ }^{11}$, H. Meyer ${ }^{37}$, J. Meyer ${ }^{10}$, S. Mikocki ${ }^{6}$, I. Milcewicz-Mika ${ }^{6}$, D. Milstead ${ }^{18}$, A. Mohamed ${ }^{18}$, F. Moreau ${ }^{29}$, A. Morozov ${ }^{8}$, J.V. Morris ${ }^{5}$, M.U. Mozer ${ }^{13}$, K. Müller ${ }^{41}$, P. Murín ${ }^{16,44}$, K. Nankov ${ }^{35}$, B. Naroska ${ }^{11}$, J. Naumann ${ }^{7}$, Th. Naumann ${ }^{39}$, P.R. Newman ${ }^{3}$, C. Niebuhr ${ }^{10}$, A. Nikiforov ${ }^{27}$, D. Nikitin ${ }^{8}$, G. Nowak $^{6}$, M. Nozicka ${ }^{33}$, R. Oganezov ${ }^{38}$, B. Olivier ${ }^{3}$, J.E. Olsson ${ }^{10}$, S. Osman ${ }^{20}$, D. Ozerov $^{25}$, C. Pascaud ${ }^{28}$, G.D. Patel ${ }^{18}$, M. Peez ${ }^{29}$, E. Perez ${ }^{9}$, D. Perez-Astudillo ${ }^{23}$, A. Perieanu ${ }^{10}$, A. Petrukhin ${ }^{25}$, D. Pitzl ${ }^{10}$, R. Plačakytè ${ }^{27}$, R. Pöschl ${ }^{10}$, B. Portheault ${ }^{28}$, B. Povh ${ }^{12}$, P. Prideaux ${ }^{18}$, N. Raicevic ${ }^{31}$, P. Reimer ${ }^{32}$, A. Rimmer ${ }^{18}$, C. Risler ${ }^{10}$, E. Rizvi ${ }^{3}$, P. Robmann ${ }^{41}$, B. Roland ${ }^{4}$, R. Roosen ${ }^{4}$, A. Rostovtsev ${ }^{25}$, Z. Rurikova $^{27}$, S. Rusakov ${ }^{26}$, F. Salvaire ${ }^{11}$, D.P.C. Sankey ${ }^{5}$, E. Sauvan ${ }^{22}$, S. Schätzel ${ }^{13}$, J. Scheins ${ }^{10}$, F.-P. Schilling ${ }^{10}$, S. Schmidt ${ }^{27}$, S. Schmitt ${ }^{41}$, C. Schmitz ${ }^{41}$, L. Schoeffel ${ }^{9}$, A. Schöning ${ }^{40}$, V. Schröder ${ }^{10}$, H.-C. Schultz-Coulon ${ }^{14}$, C. Schwanenberger ${ }^{10}$, K. Sedlák ${ }^{32}$, F. Sefkow ${ }^{10}$, I. Sheviakov ${ }^{26}$, L.N. Shtarkov ${ }^{26}$, Y. Sirois ${ }^{29}$, T. Sloan ${ }^{17}$, P. Smirnov ${ }^{26}$, Y. Soloviev ${ }^{26}$, D. South ${ }^{10}$, V. Spaskov ${ }^{8}$, A. Specka ${ }^{29}$, B. Stella ${ }^{34}$, J. Stiewe ${ }^{14}$, I. Strauch ${ }^{10}$, U. Straumann ${ }^{41}$, V. Tchoulakov ${ }^{8}$, G. Thompson ${ }^{19}$, P.D. Thompson ${ }^{3}$, F. Tomasz ${ }^{14}$, D. Traynor ${ }^{19}$, P. Truöl ${ }^{41}$, I. Tsakov ${ }^{35}$, G. Tsipolitis ${ }^{10,42}$, I. Tsurin ${ }^{10}$, J. Turnau 6 , E. Tzamariudaki ${ }^{27}$, M. Urban ${ }^{41}$, A. Usik ${ }^{26}$, D. Utkin ${ }^{25}$, S. Valkár ${ }^{33}$, A. Valkárová ${ }^{33}$, C. Vallée ${ }^{22}$, P. Van Mechelen ${ }^{4}$, N. Van Remortel ${ }^{4}$, A. Vargas Trevino ${ }^{7}$, Y. Vazdik ${ }^{26}$, C. Veelken $^{18}$, A. Vest ${ }^{1}$, S. Vinokurova ${ }^{10}$, V. Volchinski ${ }^{38}$, B. Vujicic ${ }^{27}$, K. Wacker ${ }^{7}$, J. Wagner ${ }^{10}$, G. Weber ${ }^{11}$, R. Weber ${ }^{40}$, D. Wegener ${ }^{7}$, C. Werner ${ }^{13}$, N. Werner ${ }^{41}$, M. Wessels ${ }^{1}$, B. Wessling ${ }^{10}$, C. Wigmore ${ }^{3}$, G.-G. Winter ${ }^{10}$, Ch. Wissing ${ }^{7}$, R. Wolf ${ }^{13}$, E. Wünsch ${ }^{10}$, S. Xella ${ }^{41}$, W. Yan ${ }^{10}$, V. Yeganov ${ }^{38}$, J. Žáček ${ }^{33}$, J. Zálešák ${ }^{32}$, Z. Zhang ${ }^{28}$, A. Zhelezov ${ }^{25}$, A. Zhokin ${ }^{25}$, J. Zimmermann ${ }^{27}$, H. Zohrabyan ${ }^{38}$ F. Zomer ${ }^{28}$

1 I. Physikalisches Institut der RWTH, Aachen, Germany ${ }^{\mathrm{a}}$

2 III. Physikalisches Institut der RWTH, Aachen, Germany ${ }^{a}$

3 School of Physics and Astronomy, University of Birmingham, Birmingham, $\mathrm{UK}^{\mathrm{b}}$

4 Inter-University Institute for High Energies ULB-VUB, Brussels; Universiteit Antwerpen, Antwerpen; Belgium ${ }^{c}$

5 Rutherford Appleton Laboratory, Chilton, Didcot, $\mathrm{UK}^{\mathrm{b}}$

6 Institute for Nuclear Physics, Cracow, Poland ${ }^{\mathrm{d}}$ 
7 Institut für Physik, Universität Dortmund, Dortmund, Germany ${ }^{\mathrm{a}}$

8 Joint Institute for Nuclear Research, Dubna, Russia

9 CEA, DSM/DAPNIA, CE-Saclay, Gif-sur-Yvette, France

10 DESY, Hamburg, Germany

11 Institut für Experimentalphysik, Universität Hamburg, Hamburg, Germany ${ }^{a}$

12 Max-Planck-Institut für Kernphysik, Heidelberg, Germany

13 Physikalisches Institut, Universität Heidelberg, Heidelberg, Germany ${ }^{a}$

14 Kirchhoff-Institut für Physik, Universität Heidelberg, Heidelberg, Germany ${ }^{\mathrm{a}}$

15 Institut für experimentelle und Angewandte Physik, Universität Kiel, Kiel, Germany

16 Institute of Experimental Physics, Slovak Academy of Sciences, Košice, Slovak Republic ${ }^{\mathrm{f}}$

17 Department of Physics, University of Lancaster, Lancaster, UK ${ }^{\mathrm{b}}$

18 Department of Physics, University of Liverpool, Liverpool, UK ${ }^{\mathrm{b}}$

19 Queen Mary and Westfield College, London, UK ${ }^{\mathrm{b}}$

20 Physics Department, University of Lund, Lund, Sweden ${ }^{\mathrm{g}}$

21 Physics Department, University of Manchester, Manchester, UK ${ }^{\mathrm{b}}$

22 CPPM, CNRS/IN2P3 - Univ Mediterranee, Marseille - France

23 Departamento de Fisica Aplicada, CINVESTAV, Mérida, Yucatán, México ${ }^{\mathrm{k}}$

24 Departamento de Fisica, CINVESTAV, México $^{\mathrm{k}}$

25 Institute for Theoretical and Experimental Physics, Moscow, Russia ${ }^{1}$

26 Lebedev Physical Institute, Moscow, Russia ${ }^{\text {e }}$

27 Max-Planck-Institut für Physik, München, Germany

28 LAL, Université de Paris-Sud, IN2P3-CNRS, Orsay, France

29 LLR, Ecole Polytechnique, IN2P3-CNRS, Palaiseau, France

30 LPNHE, Universités Paris VI and VII, IN2P3-CNRS, Paris, France

31 Faculty of Science, University of Montenegro, Podgorica, Serbia and Montenegro

32 Institute of Physics, Academy of Sciences of the Czech Republic, Praha, Czech Republic ${ }^{\mathrm{e}, \mathrm{i}}$

33 Faculty of Mathematics and Physics, Charles University, Praha, Czech Republic ${ }^{\mathrm{e}, \mathrm{i}}$

34 Dipartimento di Fisica Università di Roma Tre and INFN Roma 3, Roma, Italy

35 Institute for Nuclear Research and Nuclear Energy, Sofia,Bulgaria

36 Paul Scherrer Institut, Villingen, Switzerland

37 Fachbereich C, Universität Wuppertal, Wuppertal, Germany

38 Yerevan Physics Institute, Yerevan, Armenia

39 DESY, Zeuthen, Germany

40 Institut für Teilchenphysik, ETH, Zürich, Switzerland ${ }^{\mathrm{j}}$

41 Physik-Institut der Universität Zürich, Zürich, Switzerland ${ }^{\mathrm{j}}$

Received: 31 January 2005 / Revised version: 7 March 2005 /

Published online: 28 April 2005 - (C) Springer-Verlag / Società Italiana di Fisica 2005

\begin{abstract}
Measurements are reported of the production of dijet events with a leading neutron in $e p$ interactions at HERA. Differential cross sections for photoproduction and deep inelastic scattering are presented as a function of several kinematic variables. Leading order QCD simulation programs are compared with the measurements. Models in which the real or virtual photon interacts with a parton of an exchanged pion are able to describe the data. Next-to-leading order perturbative QCD calculations based on pion exchange are found to be in good agreement with the measured cross sections. The fraction of leading neutron dijet events with respect to all dijet events is also determined. The dijet events with a leading neutron have a lower fraction of resolved photon processes than do the inclusive dijet data.
\end{abstract}

\footnotetext{
42 Also at Physics Department, National Technical University, Zografou Campus, GR-15773 Athens, Greece

43 Also at Rechenzentrum, Universität Wuppertal, Wuppertal, Germany

44 Also at University of P.J. Šafárik Košice, Slovak Republic

${ }^{45}$ Also at CERN, Geneva, Switzerland

$\dagger$ Deceased

a Supported by the Bundesministerium für Bildung und Forschung, FRG, under contract numbers 05 H1 1GUA /1, 05 H1 1PAA /1, 05 H1 1PAB /9, 05 H1 1PEA /6, 05 H1 1VHA $/ 7$ and $05 \mathrm{H} 1$ 1VHB /5

b Supported by the UK Particle Physics and Astronomy Re-
}

search Council, and formerly by the UK Science and Engineering Research Council

c Supported by FNRS-FWO-Vlaanderen, IISN-IIKW and IWT and by Interuniversity Attraction Poles Programme, Belgian Science Policy

${ }^{d}$ Partially Supported by the Polish State Committee for Scientific Research, SPUB/DESY/P003/DZ 118/2003/2005

e Supported by the Deutsche Forschungsgemeinschaft

f Supported by VEGA SR grant no. 2/4067/ 24

g Supported by the Swedish Natural Science Research Council

i Supported by the Ministry of Education of the Czech Republic under the projects INGO-LA116/2000 and LN00A006, by GAUK grant no $173 / 2000$ 


\section{Introduction}

Previous HERA measurements $[1,2]$ show that the cross section for the semi-inclusive $e p$ scattering process

$$
e+p \rightarrow e+n+X
$$

where the leading neutron carries more than about $70 \%$ of the proton beam energy, is reasonably well described by the pion exchange mechanism [3-7]. In this picture, the virtual photon interacts with a parton from the pion. Constraints on the pion structure function are thus obtained. However, the Soft Colour Interaction model, in which colour neutral partonic subsystems are formed by non-perturbative soft gluon exchanges $[8,9]$, describes the data equally well [1].

In the present analysis, the leading neutron production mechanism is investigated further by requiring that the system $X$ in (1) contains two jets with large transverse momenta

$$
e+p \rightarrow e+n+j e t+j e t+X .
$$

This allows more detailed comparisons of the measurements to be made with model predictions. In addition, the jet energy provides a hard scale which allows the comparison of perturbative QCD with the data for all photon virtualities $Q^{2}$. The cross sections are measured in both photoproduction $\left(Q^{2}<10^{-2} \mathrm{GeV}^{2}\right)$ and deep inelastic scattering (DIS, $2<Q^{2}<80 \mathrm{GeV}^{2}$ ). They are given as a function of $Q^{2}$ and of the kinematic variables of the jets. Monte Carlo predictions based on leading order (LO) QCD models are compared to the data, as are next-to-leading order (NLO) QCD calculations [10]. Furthermore, a detailed comparison of dijet production with and without the requirement of a leading neutron is made. In the photoproduction regime, similar studies have been reported by the ZEUS Collaboration [11].

\section{Event kinematics and reconstruction}

The semi-inclusive reaction (1) is sketched in Fig. 1a, in which the 4-vectors of the incoming and outgoing particles and of the exchanged photon are indicated. Figure $1 \mathrm{~b}$ depicts the dijet production reaction (2) under the assumption that it is mediated by pion exchange.

The standard Lorentz invariant kinematic variables used to describe high energy $e p$ interactions are the centreof-mass energy squared $s$, the four-momentum transfer squared $Q^{2}$ and the inelasticity y:

$$
\begin{aligned}
s & \equiv(k+P)^{2}=4 E_{e} E_{p}, \\
Q^{2} & \equiv-q^{2}=-\left(k-k^{\prime}\right)^{2}=4 E_{e} E_{e}^{\prime} \cos ^{2}\left(\frac{\theta_{e}^{\prime}}{2}\right),
\end{aligned}
$$

\footnotetext{
${ }^{\mathrm{j}}$ Supported by the Swiss National Science Foundation

k Supported by CONACYT, México, grant 400073-F

${ }^{1}$ Partially Supported by Russian Foundation for Basic Research, grant no. 00-15-96584
}

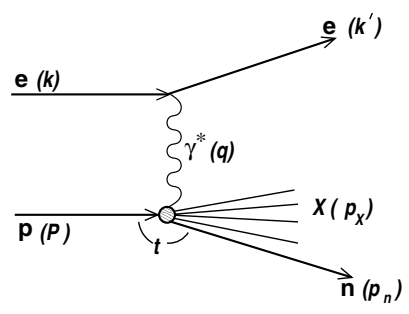

a

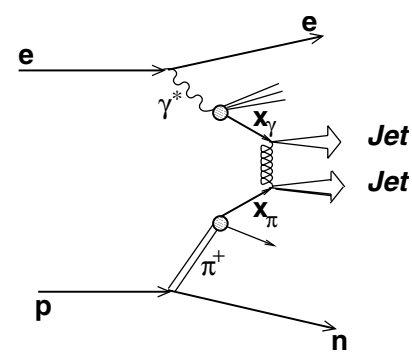

b
Fig. 1. a A generic diagram for the process $e+p \rightarrow e+n+X$, b an example diagram for the dijet production process $e+p \rightarrow$ $e+n+j e t+j e t+X$ assuming this proceeds via pion exchange

$$
y \equiv \frac{(q \cdot P)}{(k \cdot P)}=1-\frac{E_{e}^{\prime}}{E_{e}} \sin ^{2}\left(\frac{\theta_{e}^{\prime}}{2}\right) .
$$

These are determined from the energies $E_{e}$ and $E_{p}$ of the lepton and proton beams, respectively, and from the energy $E_{e}^{\prime}$ and polar angle $\theta_{e}^{\prime}$ of the scattered lepton in the laboratory frame ${ }^{1}$.

Two more invariant variables, $x_{L}$ and $t$, are used to describe the kinematics of the semi-inclusive reaction (1):

$$
\begin{aligned}
x_{L} & \equiv \frac{\left(q \cdot p_{n}\right)}{(q \cdot P)} \simeq \frac{E_{n}}{E_{p}} \\
t & \equiv\left(P-p_{n}\right)^{2} \simeq-\frac{p_{T n}^{2}}{x_{L}}-\left(1-x_{L}\right)\left(\frac{m_{n}^{2}}{x_{L}}-m_{p}^{2}\right)
\end{aligned}
$$

where $E_{n}$ is the neutron energy, $p_{T n}$ is the momentum component of the neutron transverse to the direction of the incident proton and $m_{n}$ and $m_{p}$ are the neutron and proton masses, respectively. Experimentally, $x_{L}$ and $t$ are determined from the measured energy and scattering angle of the leading neutron.

In the pion exchange model, the photon interacts with a pion emitted from the proton. In this model, process (2) is represented by diagrams as sketched in Fig. 1b. The quantity $x_{\pi}$ denotes, neglecting masses, the fraction of the 4-momentum of the pion participating in the hard interaction $^{2}$. It is related to $x_{p}$, the fraction of the 4momentum of the proton which enters the hard interaction, via $x_{p}=x_{\pi}\left(1-x_{L}\right)$.

The quantity $x_{\gamma}$ is the fraction of the 4-momentum of the photon which participates in the hard interaction. If the virtual photon is "resolved" and participates in the hard interaction via its partonic content, then $x_{\gamma}<1$. If the interactions are "direct", i.e. the entire photon enters the hard scattering process, then $x_{\gamma}=1$.

The quantities $x_{\gamma}^{\mathrm{jet}}, x_{\pi}^{\mathrm{jet}}$ and $x_{p}^{\mathrm{jet}}$, which are estimators for $x_{\gamma}, x_{\pi}$ and $x_{p}$, can be defined in dijet events using the jet transverse energies $E_{T}^{\text {jet }}$ and pseudorapidities $\eta^{\text {jet }}$

\footnotetext{
1 The right-handed $\mathrm{H} 1$ coordinate system has its positive $z$ direction along the proton beam direction and its origin at the nominal interaction point.

2 The definitions of the variables $x_{L}$ and $x_{\pi}$ are similar to the definitions of the variables $\left(1-x_{\mathbb{P}}\right)$ and $\beta$, used in $\mathrm{H} 1$ analyses of diffractive processes [12].
} 
according to:

$$
\begin{aligned}
x_{\gamma}^{\mathrm{jet}} & =\frac{\left(E_{T}^{\mathrm{jet} 1} e^{-\eta^{\mathrm{jet} 1}}+E_{T}^{\mathrm{jet} 2} e^{-\eta^{\mathrm{jet} 2}}\right)}{2 y E_{e}}, \\
x_{p}^{\mathrm{jet}} & =\frac{\left(E_{T}^{\mathrm{jet} 1} e^{\eta^{\mathrm{jet} 1}}+E_{T}^{\mathrm{jet} 2} e^{\mathrm{jet} 2}\right)}{2 E_{p}} \text { and } \\
x_{\pi}^{\mathrm{jet}} & =\frac{\left(E_{T}^{\mathrm{jet} 1} e^{\eta^{\mathrm{jet} 1}}+E_{T}^{\mathrm{jet} 2} e^{\eta^{\mathrm{jet} 2}}\right)}{2\left(E_{p}-E_{n}\right)} .
\end{aligned}
$$

The pseudorapidity is defined by $\eta=-\ln \left(\tan \frac{\theta}{2}\right)$, where $\theta$ is the polar angle with respect to the $z$ axis.

\section{Experimental procedure}

\subsection{H1 detector}

The data used in this analysis were collected with the H1 detector at HERA in the years 1996-97 and correspond to an integrated luminosity of $19.2 \mathrm{pb}^{-1}$. In these years the HERA collider was operated at positron and proton beam energies of $E_{e}=27.6 \mathrm{GeV}$ and $E_{p}=820 \mathrm{GeV}$, respectively.

A detailed description of the $\mathrm{H} 1$ detector can be found elsewhere [13]. Here only the components relevant for the present measurement are briefly described.

The $e^{+} p$ luminosity is determined with a precision of $1.6 \%$ by detecting photons from the Bethe-Heitler process $e^{+} p \rightarrow e^{+} p \gamma$ in the photon detector located at $z=-103 \mathrm{~m}$. The electron calorimeter of the luminosity system, located at $z=-33 \mathrm{~m}$, is used to detect the positrons scattered through very small angles (i.e. large $\theta_{e}^{\prime}$ ) in photoproduction events. Both detectors are $\mathrm{TlCl} / \mathrm{TlBr}$ crystal Čerenkov calorimeters with an energy resolution of $\sigma(E) / E=22 \% / \sqrt{E[\mathrm{GeV}]}$.

The Central Tracking Detector (CTD), with a polar angle coverage of $25^{\circ}<\theta<155^{\circ}$, is used to measure the trajectories of charged particles and to reconstruct the interaction vertex. The tracking system is surrounded by the finely segmented Liquid Argon (LAr) calorimeter [14], which covers the range in polar angle $4^{\circ}<\theta<154^{\circ}$ with full azimuthal acceptance. The LAr calorimeter consists of an electromagnetic section with lead as absorber, and a hadronic section with steel as absorber. The total depth of the LAr calorimeter ranges from 4.5 to 8 hadronic interaction lengths. Its energy resolution, determined in test beam measurements, is $\sigma(E) / E \approx 12 \% / \sqrt{E[\mathrm{GeV}]} \oplus 1 \%$ for electrons and $\sigma(E) / E \approx 50 \% / \sqrt{E[\mathrm{GeV}]} \oplus 2 \%$ for charged pions. The absolute electromagnetic energy scale is known with a precision of $1 \%$. The absolute hadronic energy scale for the jet energies used in this analysis is known with a precision of $4 \%$.

The polar angle region $153^{\circ}<\theta<177.8^{\circ}$ is covered by a lead/scintillating-fibre calorimeter, the SpaCal [15], with both electromagnetic and hadronic sections. For positrons, the energy resolution is $\sigma(E) / E \approx 7.1 \% / \sqrt{E[\mathrm{GeV}]} \oplus 1 \%$ and the energy scale uncertainty is less than $2 \%$. The positron polar angle is measured with a precision of $1 \mathrm{mrad}$. The hadronic energy scale in the SpaCal is known with a precision of $7 \%$. A Backward Drift Chamber (BDC) in front of the SpaCal is used to suppress background from neutral particles which can fake the scattered positron signal.

The LAr and SpaCal calorimeters are surrounded by a superconducting solenoid which provides a uniform magnetic field of $1.15 \mathrm{~T}$ along the beam direction.

Leading neutrons are detected in the Forward Neutron Calorimeter (FNC), which consists of interleaved layers of $2 \mathrm{~m}$ long lead strips and scintillator fibres and is located $107 \mathrm{~m}$ away from the nominal $\mathrm{H} 1$ interaction point in the proton beam direction (for details see [1]). The energy resolution of the calorimeter is $\sigma(E) / E \approx 20 \%$ for neutron energies between 300 and $820 \mathrm{GeV}$. The absolute hadronic energy scale is known with a precision of $5 \%$. Two segmented planes of hodoscopes situated in front of the FNC are used to veto charged particles. Each plane is constructed of $1 \mathrm{~cm}$ thick hexagonal scintillator tiles, which have the same lateral size as the calorimeter modules. The neutron detection efficiency of the FNC is $(93 \pm 5) \%$, the losses being due to the back-scattering of charged particles from the hadronic shower caused by the neutron which give signals in the veto hodoscopes. The acceptance of the FNC is defined by the aperture of the HERA beam line magnets and is limited to neutron scattering angles of $\theta_{n} \lesssim 0.8 \mathrm{mrad}$ with approximately $30 \%$ azimuthal coverage.

\subsection{Event selection}

The events used in this analysis are triggered by the coincidence of a track in the CTD with an electromagnetic cluster either in the SpaCal (DIS sample) or in the electron calorimeter of the luminosity system (photoproduction sample). A number of selection criteria are applied in order to suppress background and to confine the measurements to those regions of phase space where the acceptance is large and uniform.

The reconstructed $z$ coordinate of the event vertex is required to be within $\pm 30 \mathrm{~cm}(\sim 3 \sigma)$ of the mean $z$ position of the interaction point.

In the photoproduction analysis, the scattered positron energy $E_{e}^{\prime}$ is limited to the range $0.3<y \approx\left(1-E_{e}^{\prime} / E_{e}\right)<$ 0.65 . This cut is defined by the geometrical acceptance of the electron calorimeter which also restricts the value of $Q^{2}$ to be less than $10^{-2} \mathrm{GeV}^{2}$. To ensure that the effect of radiative corrections in photoproduction is small and to suppress events in which a Bethe-Heitler event and a photoproduction event are superimposed, the energy measured in the photon detector of the luminosity system is required to be less than $1.5 \mathrm{GeV}$.

The final state positron candidates in DIS are required to have polar scattering angles in the range $156^{\circ}<\theta_{e}^{\prime}<$ $176^{\circ}$ and energies $E_{e}^{\prime}$ greater than $10 \mathrm{GeV}$. The energy and angle, $E_{e}^{\prime}$ and $\theta_{e}^{\prime}$, are determined from the associated SpaCal cluster in combination with the interaction vertex reconstructed in the Central Tracker. The analy- 
Table 1. The kinematic regions within which the cross sections are measured

\begin{tabular}{ll}
\hline & Kinematic regions \\
\hline Photoproduction & $Q^{2}<10^{-2} \mathrm{GeV}^{2}, \quad 0.3<y<0.65$ \\
DIS & $2<Q^{2}<80 \mathrm{GeV}^{2}, \quad 0.1<y<0.7$ \\
\hline Dijets & $E_{T}^{\text {jet} 1}>7 \mathrm{GeV}, \quad E_{T}^{\text {jet2 }}>6 \mathrm{GeV}, \quad-1<\eta_{\text {lab }}^{\text {jet1,2}}<2$ \\
Neutrons & $E_{n}>500 \mathrm{GeV}, \theta_{n}<0.8 \mathrm{mrad}$ \\
\hline
\end{tabular}

sis is restricted to the region $2<Q^{2}<80 \mathrm{GeV}^{2}$ and $0.1<y<0.7$. To suppress events with initial state hard photon radiation, as well as events originating from non-ep interactions, the quantity $E-p_{z}$, summed over all reconstructed particles including the positron, is required to lie between $35 \mathrm{GeV}$ and $75 \mathrm{GeV}$. This quantity, which refers to the energy and longitudinal momentum component of each final state particle, is expected to be twice the electron beam energy for contained events.

All events that satisfy the selection cuts are subjected to a jet search using a cone algorithm with radius $R=1$ [16]. The jet finding is performed using the energies measured in the LAr and SpaCal calorimeters in the $\gamma^{*} p$ centre-of-mass frame, with transverse energies calculated relative to the $\gamma^{*}$ axis in that frame. To ensure that the bulk of the jet energy is contained within the LAr calorimeter, the laboratory pseudorapidity of each jet axis is restricted to the region $-1<\eta_{\text {lab }}^{\text {jet }}<2$. Events which have exactly two jets are selected. The transverse energies of these jets must be above $7 \mathrm{GeV}$ (first jet) and above $6 \mathrm{GeV}$ (second jet).

For the cross section measurements, events with a leading neutron are selected from the inclusive dijet samples by requiring a cluster in the FNC with an energy above $500 \mathrm{GeV}$. The background contribution due to other neutral particles is negligible at such high energies, as well as that due to dijet events overlaid with a neutron which originates from beam-gas interactions [1].

The final photoproduction data sample contains about 69000 dijet events, of which 372 events contain a leading neutron with energy $E_{\mathrm{FNC}}>500 \mathrm{GeV}$. In the DIS sample 23000 dijet events are selected, of which 213 satisfy the neutron identification criteria with $E_{\mathrm{FNC}}>500 \mathrm{GeV}$.

The kinematic regions within which this measurement is made are summarized in Table 1.

\section{Monte Carlo models}

Monte Carlo samples are used to correct the data for inefficiencies, acceptance effects, migrations and the effects of QED radiation. They are also used to correct for hadronization effects in the comparison with NLO QCD calculations. Monte Carlo predictions based on several leading order QCD models are also compared with the data.

All the Monte Carlo programs generate hard partonlevel interactions using the Born level QCD matrix elements with a minimum cut-off on the transverse momentum of the outgoing partons. They differ in the assump- tions made about the origin of the partons, which may come from either the incident proton or an exchanged pion, and in the details of the hadronization models. After hadronization, the response of the $\mathrm{H} 1$ detector to the events is simulated in detail and they are passed through the same analysis chain as is used for the data.

In addition to the models which are frequently used in studies of inclusive jet production, namely PYTHIA [17] for photoproduction and RAPGAP [18] and LEPTO [8] for DIS, models in which the hard interaction proceeds only via $\pi$-exchange are also used: the $\pi$-exchange version of RAPGAP for both photoproduction and DIS and POMPYT [19] for photoproduction. A model in which a colour neutral system is formed non-perturbatively by soft colour interactions (SCI) [9] is also compared with the data. This mechanism is implemented in the Monte Carlo program LEPTO.

The PYTHIA event generator simulates hard photonproton interactions via resolved and direct photon processes. It is used with a minimum value for the transverse momenta of the outgoing partons in the hard interaction process $\left(\hat{p}_{t}^{\mathrm{min}}\right)$ of $2 \mathrm{GeV}$. The GRV-LO parton densities are used for the photon [20] and the proton [21]. The photon flux is calculated in the Weizsäcker-Williams approximation [22]. Higher order QCD radiation effects are simulated using initial and final state parton showers in the leading $\log$ approximation. The subsequent fragmentation follows the Lund string model as implemented in JETSET 7.4 [23]. PYTHIA can also simulate multi-parton interactions (MI), which are calculated as LO QCD processes between partons from the remnants of the proton and the resolved photon. The resulting additional final state partons are required to have transverse momenta above a cut-off value of $1.2 \mathrm{GeV}$. It has previously been shown [24] that these additional interactions improve considerably the description of inclusive jet photoproduction. This option of PYTHIA is referred to as PYTHIA-MI below. The PYTHIA calculation is performed with version 5.7 and cross-checked with version 6.1 .

The program LEPTO 6.5 [8] generates DIS events. It is based on leading order electroweak cross sections and takes QCD effects into account to order $\alpha_{s}$. As in PYTHIA, higher order QCD effects are simulated using leading log parton showers and the final state hadrons are obtained via Lund string fragmentation. Higher order electroweak processes are simulated using DJANGO [25], an interface between LEPTO and HERACLES [26]. The LEPTO program allows the simulation of soft colour interactions [9], through which the production of leading baryons and diffraction-like configurations is enhanced via 
non-perturbative colour rearrangements between the outgoing partons. In the following, the predictions based on this approach are denoted LEPTO-SCI.

The program RAPGAP 2.8 [18] is a general purpose event generator for inclusive and diffractive $e p$ interactions. In DIS, the RAPGAP simulation includes a contribution from resolved photon events in which the photon structure is parameterized according to the SaS-2D [27] parton densities. These give a reasonable description of inclusive dijet production at low $Q^{2}$ [28]. In the version denoted below as RAPGAP- $\pi$, the program simulates exclusively the scattering of virtual or real photons off an exchanged pion. Here, the cross section for photon-proton scattering to the final state $n X$ takes the form

$$
d \sigma^{\gamma^{*} p \rightarrow n X}=f_{\pi^{+} / p}\left(x_{L}, t\right) \cdot d \sigma^{\gamma^{*} \pi^{+} \rightarrow X},
$$

where $f_{\pi^{+} / p}\left(x_{L}, t\right)$ is the pion flux associated with the beam proton and $d \sigma^{\gamma^{*} \pi^{+} \rightarrow X}$ denotes the cross section for the hard photon-pion interaction. Several pion flux parameterizations can be found in the literature. However, a detailed study of different fluxes is not possible with the present data. In this measurement, the flux factor is therefore taken from [4], which is the default parameterization in the RAPGAP program and which provides a reasonable description of the experimental control distributions. It is defined as

$$
\begin{aligned}
f_{\pi^{+} / p}\left(x_{L}, t\right)= & \frac{1}{2 \pi} \frac{g_{p \pi n}^{2}}{4 \pi}\left(1-x_{L}\right) \frac{-t}{\left(m_{\pi}^{2}-t\right)^{2}} \\
& \times \exp \left(-R_{\pi n}^{2} \frac{m_{\pi}^{2}-t}{1-x_{L}}\right),
\end{aligned}
$$

where $m_{\pi}$ is the pion mass, $g_{p \pi n}^{2} / 4 \pi=13.6$ is the $p \pi n$ coupling constant, known from phenomenological analyses of low-energy data [29], and $R_{\pi n}=0.93 \mathrm{GeV}^{-1}$ is the radius of the pion-neutron Fock state of the proton [4]. For the range of $t$ and $x_{L}$ relevant here $\left(-t \lesssim 0.6 \mathrm{GeV}^{2}, x_{L} \gtrsim 0.5\right)$ this flux parameterization yields results very similar to those of the parameterization of [5], which includes the full $t$ dependence expected from the pion Regge trajectory and which is used in our previous analysis [1]. In the kinematic range of the measurements, leading neutron production due to $\rho$ and $a_{2}$ exchanges, to pomeron exchange, and to resonance decays, is one order of magnitude smaller than the contribution due to pion exchange $[1,5]$. If not otherwise stated, the GRV- $\pi$-LO [30] pion structure function parameterization is used.

The POMPYT 2.6 [19] Monte Carlo program is an extension of PYTHIA, which models colour singlet exchange processes in photoproduction. For pion exchange processes, POMPYT simulates the scattering of real photons off the exchanged pion, using the pion flux parameterization of (7). POMPYT yields results very similar to those of RAPGAP- $\pi$.

\section{Systematic uncertainties}

The acceptance of the FNC calorimeter is defined by the interaction point and the geometry of the beam guiding magnets and is determined using Monte Carlo simulations. The angular distribution of the neutrons produced in reaction (1) is sharply peaked in the forward direction, and the observed cross section therefore depends critically on small inclinations of the incoming proton beam with respect to its nominal direction. This effect is studied with the help of the Monte Carlo simulations described above. The overall uncertainty in the FNC acceptance is estimated to be about $10 \%$. The uncertainty in the neutron detection efficiency leads to an additional $5 \%$ systematic error, and the uncertainty of the FNC absolute energy scale to a $6 \%$ systematic error. The uncertainties due to the background from other neutral particles and due to the overlap of dijet events with beam-gas interactions are less than $1 \%$. These effects contribute to the overall normalization error.

The $4 \%$ uncertainty on the absolute hadronic energy scale of the LAr calorimeter leads to an uncertainty of about $15 \%$ on the jet cross section. This is strongly correlated between data points.

The uncertainty of the acceptance of the electron calorimeter of the luminosity system, in the photoproduction case, amounts to about $6 \%$. This includes the uncertainty on the luminosity measurement of $1.6 \%$ and contributes to the overall normalization error.

The uncertainties on the measurements of the positron energy and angle in the SpaCal lead to $6 \%$ systematic uncertainties in the DIS cross sections.

As shown below, the models based on the pionexchange mechanism describe the data well and are therefore used to estimate acceptance and migration corrections. These corrections are determined from the POMPYT Monte Carlo simulations in the photoproduction case and using RAPGAP- $\pi$ for DIS. The uncertainties of the corrections are estimated from the differences in the results when other models are used: PYTHIA or RAPGAP- $\pi$ in the photoproduction case and LEPTO or RAPGAP in the DIS case. The estimated uncertainties are between $10 \%$ and $15 \%$ for all distributions.

Due to the energy cut in the photon detector, QED radiative corrections are small [31] in the photoproduction case and are neglected here. For the DIS sample, the QED radiative corrections amount to less than $10 \%$, as evaluated using RAPGAP interfaced to the HERACLES program. The uncertainty arising from the radiative corrections is about $5 \%$.

Finally, a $3 \%$ correlated uncertainty is attributed to the trigger efficiencies as evaluated using other, independent triggers.

The correlated error contributions are only weakly dependent on the kinematic variables studied, causing a normalization uncertainty of about $20 \%$ on the cross sections in both the DIS and photoproduction cases. The uncorrelated, point-to-point systematic uncertainties range from $11 \%$ to $17 \%$.

In the figures, the outer error bars represent the quadratic sum of the point-to-point systematic errors and the statistical errors, while the inner error bars show the statistical errors. The normalization error is not shown in 

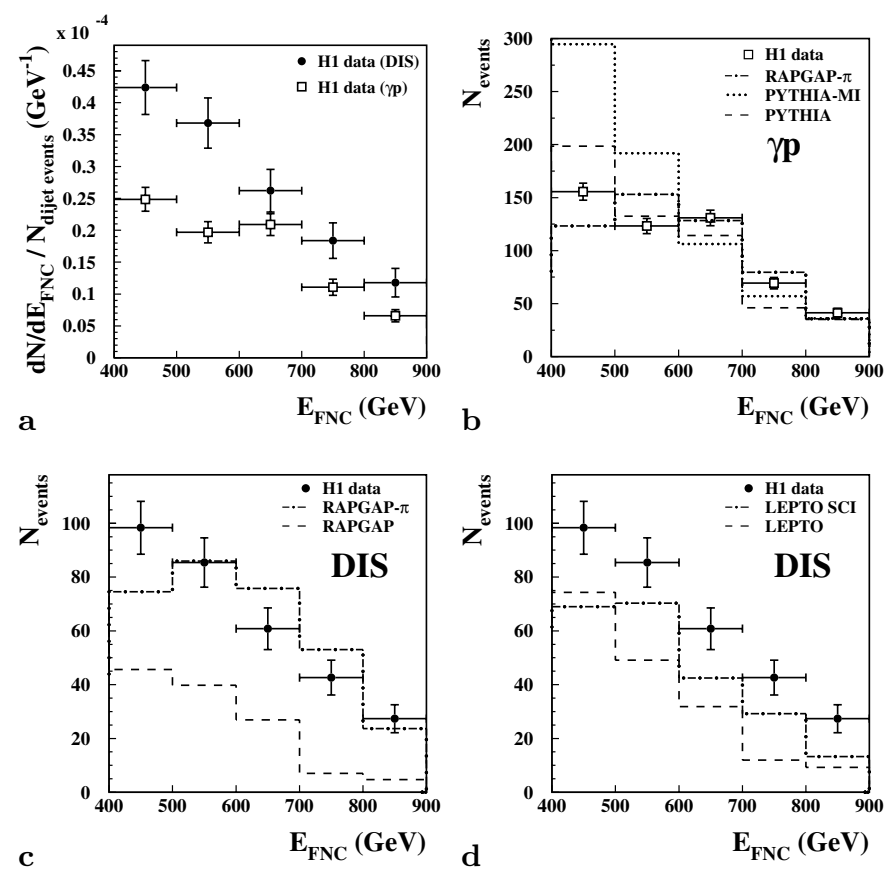

Fig. 2. Energy distributions observed in the FNC for dijet events: a Photoproduction data compared with DIS data, normalized to the respective number of dijet events in each inclusive dijet sample; b The Monte Carlo model predictions for RAPGAP- $\pi$, PYTHIA and PYTHIA-MI, compared with photoproduction data; c, d The Monte Carlo models RAPGAP (both the $\pi$-exchange and the standard DIS versions) and LEPTO (with and without SCI), compared with DIS data. The errors on the data points are statistical only. The corresponding kinematic regions for the photoproduction, DIS and dijet selections are given in Table 1

the figures, but is included in the tables as a correlated systematic error.

\section{Results and discussion}

\subsection{Neutron energy distribution}

The neutron energy spectrum allows discrimination between different models for the production of leading neutron events. Figure 2 shows the energy spectra for the photoproduction and DIS dijet samples, as measured in the FNC, for energies above $400 \mathrm{GeV}$. The data are not corrected for efficiencies, acceptance or migration between bins. These effects, however, are taken into account in the Monte Carlo simulations. In Fig. 2a the photoproduction and DIS data are shown, normalized to the total number of events in the corresponding inclusive dijet samples. There is a significantly higher fraction of leading neutrons in DIS dijet events than in photoproduction dijet events, especially for the lowest neutron energies. However, the shape of the energy spectrum is similar in both samples.

In Figs. 2b-d the photoproduction and DIS data are shown together with the predictions from several Monte Carlo models. The model predictions are all normalized to the integrated luminosity of the corresponding data samples.

For simulated neutron energies $E_{n}>400 \mathrm{GeV}$, the photoproduction data in Fig. $2 \mathrm{~b}$ are reasonably well described in shape and magnitude by the $\pi$-exchange model RAPGAP- $\pi$, as well as by PYTHIA without multiple interactions. If multiple interactions are included, PYTHIA fails to describe the data, predicting a rate which is too high for neutron energies between 400 and $600 \mathrm{GeV}$. This observation is in contrast to the inclusive jet measurement (without the requirement of a leading neutron), which is described by PYTHIA only if multiple interactions are included, especially at low jet $E_{T}$ [24].

The RAPGAP predictions are compared with DIS data in Fig. 2c. The $\pi$-exchange version describes the shape of the distribution well, but somewhat overestimates the absolute rate, while the rate predicted by the standard RAPGAP DIS version is too low. The LEPTO prediction is also too low, as is shown in Fig. 2d. The LEPTO predictions are somewhat increased for $E_{n} \gtrsim 500 \mathrm{GeV}$ if soft colour interactions (LEPTO-SCI) are included.

To summarize, the pion exchange models describe the shape of the observed FNC energy spectra well for energies above $500 \mathrm{GeV}$, as does LEPTO with soft colour interactions.

\subsection{Cross section measurements}

In this section, differential cross sections at the hadron level are presented for dijet production in the photoproduction and DIS regimes for neutron energies $E_{n}>$ $500 \mathrm{GeV}$, corresponding to the region in which $\pi$-exchange models give a good description of the $E_{\mathrm{FNC}}$ distribution. The data are corrected for detector inefficiencies and migrations due to detector resolution effects using the Monte Carlo simulations described in Sects. 4 and 5. The results are given in Figs. 3-6 and Tables 2-3.

In Fig. 3 the jet cross sections are shown as a function of $E_{T}^{\text {jet }}$ and $\eta_{\text {lab }}^{\text {jet }}$ for the photoproduction and DIS regimes using both jets in the event.

Taking the $20 \%$ normalization uncertainty into account, the data are well described by the $\pi$-exchange model RAPGAP- $\pi$ in both DIS and photoproduction. However, PYTHIA without multiple interactions, which does not include pion exchange, also provides a good description of the photoproduction data. The inclusion of multiple interactions in PYTHIA results in a predicted cross section which is too high for low values of $E_{T}^{\text {jet }}$ and for values of $\eta_{\text {lab }}^{\text {jet }}>-0.5$. It is also seen from Figs. $3 b$ and 3d that standard DIS processes, as simulated by the LEPTO program, tend to lie below the data, even if soft colour interactions are included.

The measured $Q^{2}$ dependence of the dijet DIS cross section is shown in Fig. 4, together with predictions from Monte Carlo simulations. The $\pi$-exchange version of RAPGAP describes the measured distribution fairly well, whereas LEPTO with SCI reproduces the shape of the 

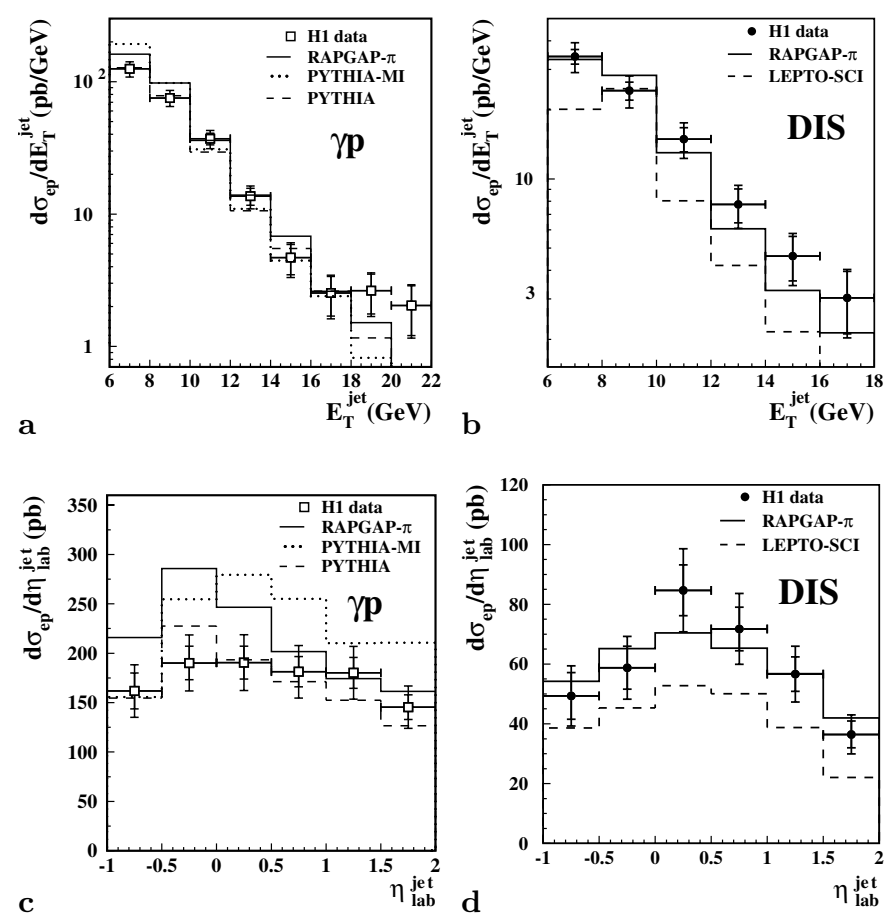

Fig. 3. The measured differential $e p$ cross sections as a function of $E_{T}^{\text {jet }}$ and $\eta_{\text {lab }}^{\text {jet }}$ for dijet events with a leading neutron. The cross section is given for photoproduction a,c and DIS $\mathbf{b}, \mathbf{d}$ and compared with the Monte Carlo simulations. Inner error bars show the statistical errors, while the outer error bars represent the statistical and systematic errors, added in quadrature. The overall normalization uncertainty of $20 \%$ is not shown. The kinematic regions within which this measurement is made are given in Table 1

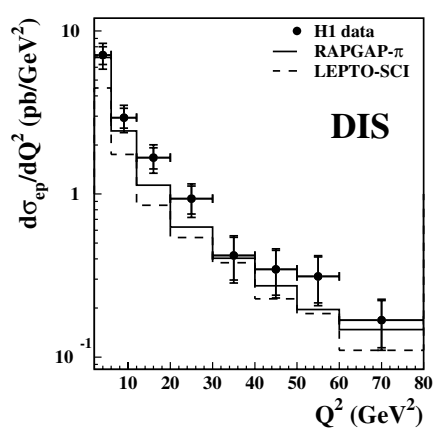

Fig. 4. The measured deep inelastic ep cross section as a function of $Q^{2}$ for dijet events with a leading neutron. Inner error bars show the statistical errors, while the outer error bars represent the statistical and systematic errors, added in quadrature. The overall normalization uncertainty of $20 \%$ is not shown. Predictions from Monte Carlo simulations are compared with the measurements. The kinematic regions within which this measurement is made are given in Table 1

distribution but yields a lower prediction over the whole $Q^{2}$ range.

The dependence of the dijet cross section on the fractional momenta $x_{\gamma}^{\text {jet }}$ and $x_{\pi}^{\text {jet }}$, determined according to (5), is shown in Figs. 5 and 6. The measured $x_{\gamma}^{\text {jet }}$ dis-
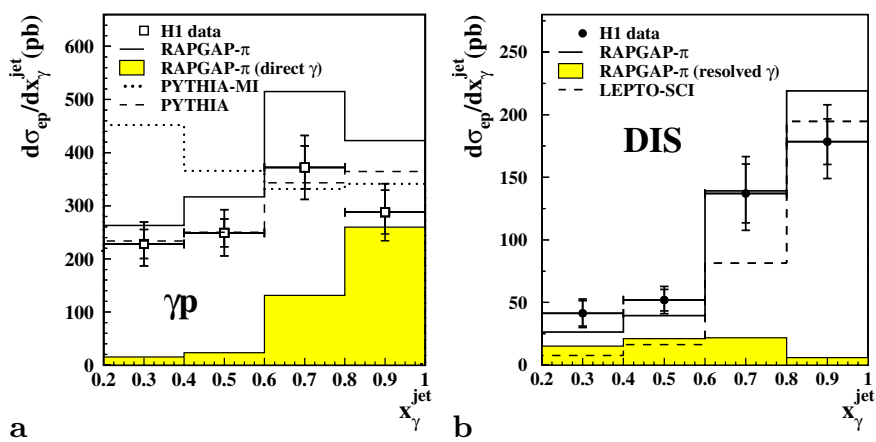

Fig. 5. The measured differential $e p$ cross sections as a function of $x_{\gamma}^{\text {jet }}$ for dijet events with a leading neutron. The cross section is given for photoproduction $\mathbf{a}$ and DIS $\mathbf{b}$ and compared with the Monte Carlo simulations. Inner error bars show the statistical errors, while the outer error bars represent the statistical and systematic errors, added in quadrature. The overall normalization uncertainty of $20 \%$ is not shown. The kinematic regions within which this measurement is made are given in Table 1
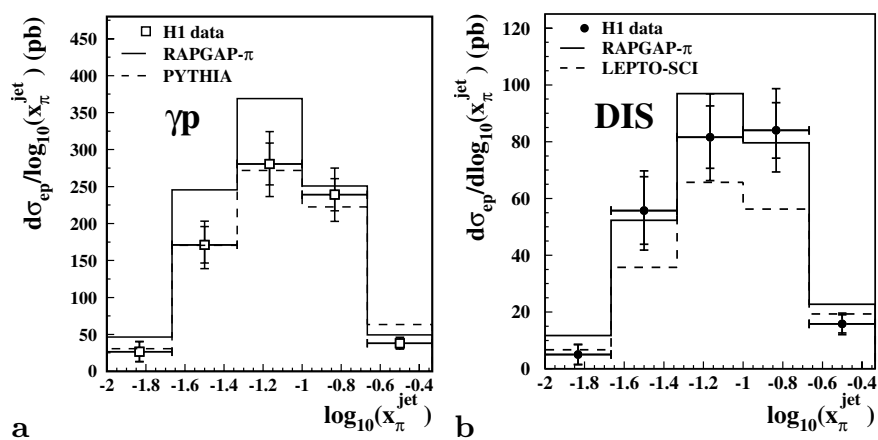

Fig. 6. The measured differential $e p$ cross sections as a function of $x_{\pi}^{\text {jet }}$ for dijet events with a leading neutron. The cross section is given for photoproduction $\mathbf{a}$ and DIS $\mathbf{b}$ and compared with the Monte Carlo simulations. Inner error bars show the statistical errors, while the outer error bars represent the statistical and systematic errors, added in quadrature. The overall normalization uncertainty of $20 \%$ is not shown. The kinematic regions within which this measurement is made are given in Table 1

tribution in the photoproduction regime, together with the RAPGAP- $\pi$ and PYTHIA model predictions shown in Fig. 5a, clearly demonstrate the large contribution of resolved photon processes. The shape of the distribution is well described by PYTHIA and RAPGAP- $\pi$. PYTHIA with multiple interactions predicts too high a cross section at $x_{\gamma}^{\text {jet }}<0.6$. In the DIS regime, as is clear from the $x_{\gamma}^{\text {jet }}$ distribution shown in Fig. 5b, direct photon interactions dominate. However, a small fraction $(\sim 15 \%)$ of resolved photon interactions is necessary to fully describe the data with the RAPGAP- $\pi$ simulation. The LEPTO-SCI model, which does not include resolved photon processes, provides a poor description of the shape of the distributions.

The $x_{\pi}^{\text {jet }}$ distributions in the photoproduction and the DIS samples, shown in Figs. 6a and 6b, respectively, are similar in shape. The $\pi$-exchange model RAPGAP- $\pi$, and 
Table 2. The differential $e p$ photoproduction cross section as a function of $E_{T}^{\text {jet }}, \eta_{\text {lab }}^{\text {jet }}, x_{\gamma}^{\text {jet }}$ and $x_{\pi}^{\text {jet }}$ for dijet events with a leading neutron. The kinematic regions within which this measurement is made are given in Table 1

\begin{tabular}{|c|c|c|c|c|c|c|}
\hline \multicolumn{3}{|c|}{$\begin{array}{c}\text { Jet transverse energy }\left(E_{T}^{\text {jet }}\right) \\
{[\mathrm{GeV}]}\end{array}$} & $\begin{array}{c}d \sigma_{e p} / d E_{T}^{\mathrm{jet}} \\
{[\mathrm{pb} / \mathrm{GeV}]} \\
\end{array}$ & $\begin{array}{c}\delta_{\text {stat. }} \\
{[\mathrm{pb} / \mathrm{GeV}]}\end{array}$ & $\begin{array}{c}\delta_{\text {uncorrel.syst. }} \\
{[\mathrm{pb} / \mathrm{GeV}]}\end{array}$ & $\begin{array}{l}\delta_{\text {correl.syst. }} \\
{[\mathrm{pb} / \mathrm{GeV}]}\end{array}$ \\
\hline 6 & - & 8 & 125. & 7. & 15. & 25. \\
\hline 8 & - & 10 & 75.4 & 5.2 & 9.1 & 15.1 \\
\hline 10 & - & 12 & 37.1 & 3.7 & 4.5 & 7.4 \\
\hline 12 & - & 14 & 13.6 & 2.0 & 1.8 & 2.7 \\
\hline 14 & - & 16 & 4.69 & 1.21 & 0.65 & 0.94 \\
\hline 16 & - & 18 & 2.52 & 0.84 & 0.37 & 0.51 \\
\hline 18 & - & 20 & 2.63 & 0.88 & 0.38 & 0.53 \\
\hline 20 & - & 22 & 2.04 & 0.83 & 0.30 & 0.41 \\
\hline \multicolumn{3}{|c|}{ Jet pseudorapidity $\left(\eta_{\text {lab }}^{\text {jet }}\right)$} & $\begin{array}{c}d \sigma_{e p} / d \eta_{\text {lab }}^{\text {jet }} \\
{[\mathrm{pb}]}\end{array}$ & $\begin{array}{r}\delta_{\text {stat. }} \\
{[\mathrm{pb}]}\end{array}$ & $\begin{array}{c}\delta_{\text {uncorrel.syst. }} \\
{[\mathrm{pb}]}\end{array}$ & $\begin{array}{c}\delta_{\text {correl.syst. }} \\
{[\mathrm{pb}]}\end{array}$ \\
\hline-1.0 & - & -0.5 & 162. & 18. & 19. & 32. \\
\hline-0.5 & - & 0.0 & 190. & 17. & 23. & 38. \\
\hline 0.0 & - & 0.5 & 191. & 17. & 23. & 38. \\
\hline 0.5 & - & 1.0 & 181. & 15. & 22. & 36. \\
\hline 1.0 & - & 1.5 & 180. & 16. & 22. & 36. \\
\hline 1.5 & - & 2.0 & 145. & 13. & 17. & 29. \\
\hline \multicolumn{3}{|c|}{$x_{\gamma}^{\text {jet }}$} & $\begin{array}{c}d \sigma_{e p} / d x_{\gamma}^{\mathrm{jet}} \\
{[\mathrm{pb}]}\end{array}$ & $\begin{array}{c}\delta_{\text {stat. }} \\
{[\mathrm{pb}]}\end{array}$ & $\begin{array}{c}\delta_{\text {uncorrel.syst. }} \\
{[\mathrm{pb}]}\end{array}$ & $\begin{array}{c}\delta_{\text {correl.syst. }} \\
{[\mathrm{pb}]}\end{array}$ \\
\hline 0.2 & - & 0.4 & 228. & 27. & 31. & 46. \\
\hline 0.4 & - & 0.6 & 249. & 26. & 35. & 50. \\
\hline 0.6 & - & 0.8 & 372. & 40. & 45. & 74. \\
\hline 0.8 & - & 1.0 & 288. & 41. & 35. & 58. \\
\hline \multicolumn{3}{|c|}{$\log _{10}\left(x_{\pi}^{\text {jet }}\right)$} & $\begin{array}{c}d \sigma_{e p} / d \log _{10}\left(x_{\pi}^{\text {jet }}\right) \\
{[\mathrm{pb}]}\end{array}$ & $\begin{array}{l}\delta_{\text {stat. }} \\
{[\mathrm{pb}]}\end{array}$ & $\begin{array}{c}\delta_{\text {uncorrel.syst. }} \\
{[\mathrm{pb}]}\end{array}$ & $\begin{array}{c}\delta_{\text {correl.syst. }} \\
{[\mathrm{pb}]}\end{array}$ \\
\hline-2.00 & - & -1.67 & 26.6 & 13.3 & 4.0 & 5.3 \\
\hline-1.67 & - & -1.33 & 171. & 25. & 21. & 34. \\
\hline-1.33 & - & -1.00 & 281. & 28. & 34. & 56. \\
\hline-1.00 & - & -0.67 & 239. & 22 . & 29. & 48. \\
\hline-0.67 & - & -0.33 & 38.1 & 5.6 & 5.7 & 7.6 \\
\hline
\end{tabular}

the PYTHIA and LEPTO-SCI models, provide a fair description of the data.

For the RAPGAP- $\pi$ predictions shown in Figs. 3 to 6 the GRV- $\pi$-LO parameterization of the pion parton distribution functions (PDFs) is used. However, within the $20 \%$ normalization uncertainty, a similar quality of description is provided if other parameterizations of the pion PDFs $[32,33]$ are used.

\subsection{Comparison of the photoproduction cross section with NLO QCD}

The parton level cross sections for dijet photoproduction in photon-pion and photon-proton collisions are calculated in QCD at both leading and next-to-leading order [10]. In the latter case, infrared and collinear singularities are cancelled using the phase space slicing method with an invariant mass cut-off. The renormalization and factorization scales are defined to be the maximum transverse energy of the outgoing partons. The scale uncertainty amounts to approximately $15 \%$ on average, but is significantly larger (up to $30 \%$ ) for low $x_{\gamma}^{\text {jet }}$ and high $\eta_{\text {lab }}^{\text {jet }}$, as estimated by varying the scales by factors of 0.5 and 2. The photon flux is calculated using the WeizsäckerWilliams approximation [22]. The light-cone form factor [4] is used in the pion flux with the same parameters as for the RAPGAP- $\pi$ Monte Carlo predictions. In the calculations, the GRV parameterizations for the parton distribution functions are used for both the photon and the pion. A cone algorithm with radius $R=1$ is used in the definition of jets.

Since the QCD calculations refer to jets of partons, whereas the measurements refer to jets of hadrons, the predicted cross sections are corrected to the hadron level using factors evaluated from the LO Monte Carlo pro- 
Table 3. The differential deep inelastic ep cross section as a function of $E_{T}^{\text {jet }}, \eta_{\text {lab }}^{\text {jet }}, x_{\gamma}^{\text {jet }}, x_{\pi}^{\text {jet }}$ and $Q^{2}$ for dijet events with a leading neutron. The kinematic regions within which this measurement is made are given in Table 1

\begin{tabular}{|c|c|c|c|c|c|}
\hline \multicolumn{2}{|c|}{$\begin{array}{c}\text { Jet transverse energy }\left(E_{T}^{\text {jet }}\right) \\
{[\mathrm{GeV}]}\end{array}$} & $\begin{array}{c}d \sigma_{e p} / d E_{T}^{\mathrm{jet}} \\
{[\mathrm{pb} / \mathrm{GeV}]}\end{array}$ & $\begin{array}{c}\delta_{\text {stat. }} \\
{[\mathrm{pb} / \mathrm{GeV}]}\end{array}$ & $\begin{array}{c}\delta_{\text {uncorrel.syst. }} \\
{[\mathrm{pb} / \mathrm{GeV}]}\end{array}$ & $\begin{array}{l}\delta_{\text {correl.syst. }} \\
{[\mathrm{pb} / \mathrm{GeV}]}\end{array}$ \\
\hline 6 & 8 & 32.2 & 2.5 & 4.3 & 6.4 \\
\hline 8 & 10 & 24.4 & 2.2 & 3.3 & 4.9 \\
\hline 10 & 12 & 14.2 & 1.8 & 1.9 & 2.8 \\
\hline 12 & 14 & 7.8 & 1.3 & 1.1 & 1.6 \\
\hline 14 & 16 & 4.53 & 1.01 & 0.66 & 0.91 \\
\hline 16 & 18 & 2.90 & 0.92 & 0.43 & 0.58 \\
\hline \multicolumn{2}{|c|}{ Jet pseudorapidity $\left(\eta_{\text {lab }}^{\text {jet }}\right)$} & $\begin{array}{c}d \sigma_{e p} / d \eta_{\text {lab }}^{\text {jet }} \\
{[\mathrm{pb}]}\end{array}$ & $\begin{array}{l}\delta_{\text {stat. }} \\
{[\mathrm{pb}]}\end{array}$ & $\begin{array}{c}\delta_{\text {uncorrel.syst. }} \\
{[\mathrm{pb}]}\end{array}$ & $\begin{array}{c}\delta_{\text {correl.syst. }} \\
{[\mathrm{pb}]}\end{array}$ \\
\hline-1 & -0.5 & 45.3 & 7.8 & 6.1 & 9.1 \\
\hline-0.5 & 0.0 & 58.1 & 7.2 & 7.8 & 11.6 \\
\hline 0.0 & 0.5 & 83.2 & 8.5 & 11.1 & 16.6 \\
\hline 0.5 & 1.0 & 69.7 & 7.3 & 9.3 & 13.9 \\
\hline 1.0 & 1.5 & 53.9 & 5.8 & 7.2 & 10.8 \\
\hline 1.5 & 2.0 & 33.1 & 4.5 & 4.4 & 6.6 \\
\hline \multicolumn{2}{|c|}{$x_{\gamma}^{\text {jet }}$} & $\begin{array}{c}d \sigma_{e p} / d x_{\gamma}^{\text {jet }} \\
{[\mathrm{pb}]}\end{array}$ & $\begin{array}{c}\delta_{\text {stat. }} \\
{[\mathrm{pb}]}\end{array}$ & $\begin{array}{c}\delta_{\text {uncorrel.syst. }} \\
{[\mathrm{pb}]}\end{array}$ & $\begin{array}{c}\delta_{\text {correl.syst. }} \\
{[\mathrm{pb}]}\end{array}$ \\
\hline 0.2 & 0.4 & 35.0 & 10.1 & 5.3 & 7.0 \\
\hline 0.4 & 0.6 & 40.8 & 8.6 & 6.2 & 8.2 \\
\hline 0.6 & 0.8 & 161. & 24. & 22. & 32. \\
\hline 0.8 & 1.0 & 190. & 18. & 25. & 38. \\
\hline \multicolumn{2}{|c|}{$\log _{10}\left(x_{\pi}^{\text {jet }}\right)$} & $\begin{array}{c}d \sigma_{e p} / d \log _{10}\left(x_{\pi}^{\mathrm{jet}}\right) \\
{[\mathrm{pb}]}\end{array}$ & $\begin{array}{l}\delta_{\text {stat. }} \\
{[\mathrm{pb}]}\end{array}$ & $\begin{array}{c}\delta_{\text {uncorrel.syst. }} \\
{[\mathrm{pb}]}\end{array}$ & $\begin{array}{c}\delta_{\text {correl.syst. }} \\
{[\mathrm{pb}]}\end{array}$ \\
\hline-2.00 & -1.67 & 5.0 & 3.5 & 0.8 & 1.0 \\
\hline-1.67 & -1.33 & 56. & 12. & 8. & 11. \\
\hline-1.33 & -1.00 & 82. & 11. & 11. & 16. \\
\hline-1.00 & -0.67 & 84. & 10. & 12. & 17. \\
\hline-0.67 & -0.33 & 15.8 & 3.2 & 2.4 & 3.2 \\
\hline \multicolumn{2}{|c|}{$\begin{array}{c}Q^{2} \\
{\left[\mathrm{GeV}^{2}\right]}\end{array}$} & $\begin{array}{c}d \sigma_{e p} / d Q^{2} \\
{\left[\mathrm{pb} / \mathrm{GeV}^{2}\right]}\end{array}$ & $\begin{array}{c}\delta_{\text {stat. }} \\
{\left[\mathrm{pb} / \mathrm{GeV}^{2}\right]}\end{array}$ & $\begin{array}{c}\delta_{\text {uncorrel.syst. }} \\
{\left[\mathrm{pb} / \mathrm{GeV}^{2}\right]}\end{array}$ & $\begin{array}{l}\delta_{\text {correl.syst. }} \\
{\left[\mathrm{pb} / \mathrm{GeV}^{2}\right]}\end{array}$ \\
\hline 2 & 6 & 7.06 & 0.88 & 0.94 & 1.40 \\
\hline 6 & 12 & 2.82 & 0.41 & 0.38 & 0.56 \\
\hline 12 & 20 & 1.51 & 0.25 & 0.20 & 0.30 \\
\hline 20 & 30 & 0.95 & 0.18 & 0.13 & 0.19 \\
\hline 30 & 40 & 0.408 & 0.123 & 0.059 & 0.082 \\
\hline 40 & 50 & 0.319 & 0.106 & 0.046 & 0.064 \\
\hline 50 & 60 & 0.277 & 0.098 & 0.042 & 0.055 \\
\hline 60 & 80 & 0.153 & 0.054 & 0.023 & 0.031 \\
\hline
\end{tabular}

grams described in Sect. 4. The hadronization correction factor, $\left(1+\delta_{h a d r}\right)$, is defined as the ratio of the cross section obtained with jets reconstructed from hadrons to that using jets reconstructed at the parton level after the generation of parton showers. The corrections are calculated by taking an average of the results from two different Monte Carlo models (POMPYT and RAPGAP- $\pi$ ). The uncertainty of these corrections is taken to be half the difference between the results obtained from the two models, which is typically smaller than $5 \%$. The hadronization corrections have a tendency to increase the calculated NLO cross section at low $E_{T}^{\text {jet }}$ (by approximately $30 \%$ for $E_{T}^{\text {jet }}=7 \mathrm{GeV}$ ) and to decrease the cross section at high $E_{T}^{\text {jet }}$ (by approximately $-10 \%$ for $\left.E_{T}^{\mathrm{jet}}=20 \mathrm{GeV}\right)$. As a function of $x_{\gamma}^{\mathrm{jet}}$, the hadronization corrections increase the cross section by about $25 \%$ at the lowest $x_{\gamma}^{\text {jet }}$, by about $100 \%$ for $x_{\gamma}^{\text {jet }}=0.8$, 
Table 4. The ratio of the cross section for dijet photoproduction with a leading neutron to that for inclusive dijet photoproduction, as a function of $E_{T}^{\text {jet }}, \eta_{\text {lab }}^{\text {jet }}, x_{\gamma}^{\text {jet }}$ and $x_{p}^{\text {jet }}$. The kinematic regions within which this measurement is made are given in Table 1

\begin{tabular}{|c|c|c|c|c|c|c|}
\hline \multicolumn{3}{|c|}{$\begin{array}{c}\text { Jet transverse energy }\left(E_{T}^{\mathrm{jet}}\right) \\
{[\mathrm{GeV}]}\end{array}$} & $f_{l n}$ & $\delta_{\text {stat. }}$ & $\delta_{\text {uncorrel.syst. }}$ & $\delta_{\text {correl.syst. }}$ \\
\hline 6 & - & 8 & 0.0231 & 0.0012 & 0.0026 & 0.0030 \\
\hline 8 & - & 10 & 0.0244 & 0.0017 & 0.0027 & 0.0031 \\
\hline 10 & - & 12 & 0.0200 & 0.0020 & 0.0032 & 0.0025 \\
\hline 12 & - & 14 & 0.0202 & 0.0030 & 0.0032 & 0.0026 \\
\hline 14 & - & 16 & 0.0212 & 0.0055 & 0.0033 & 0.0027 \\
\hline 16 & - & 18 & 0.0152 & 0.0050 & 0.0024 & 0.0019 \\
\hline \multicolumn{3}{|c|}{ Jet pseudorapidity $\left(\eta_{\text {lab }}^{\text {jet }}\right)$} & $f_{l n}$ & $\delta_{\text {stat. }}$ & $\delta_{\text {uncorrel.syst. }}$ & $\delta_{\text {correl.syst. }}$ \\
\hline-1.0 & - & -0.5 & 0.0363 & 0.0041 & 0.0041 & 0.0046 \\
\hline-0.5 & - & 0.0 & 0.0277 & 0.0025 & 0.0031 & 0.0035 \\
\hline 0.0 & - & 0.5 & 0.0221 & 0.0019 & 0.0027 & 0.0028 \\
\hline 0.5 & - & 1.0 & 0.0218 & 0.0018 & 0.0027 & 0.0028 \\
\hline 1.0 & - & 1.5 & 0.0211 & 0.0018 & 0.0027 & 0.0027 \\
\hline 1.5 & - & 2.0 & 0.0143 & 0.0012 & 0.0018 & 0.0018 \\
\hline \multicolumn{3}{|c|}{$x_{\gamma}^{\text {jet }}$} & $f_{l n}$ & $\delta_{\text {stat. }}$ & $\delta_{\text {uncorrel.syst. }}$ & $\delta_{\text {correl.syst. }}$ \\
\hline 0.2 & - & 0.4 & 0.0105 & 0.0013 & 0.0013 & 0.0013 \\
\hline 0.4 & - & 0.6 & 0.0256 & 0.0027 & 0.0029 & 0.0033 \\
\hline 0.6 & - & 0.8 & 0.0340 & 0.0037 & 0.0038 & 0.0044 \\
\hline 0.8 & - & 1.0 & 0.0344 & 0.0049 & 0.0039 & 0.0045 \\
\hline \multicolumn{3}{|c|}{$\log _{10}\left(x_{p}^{\text {jet }}\right)$} & $f_{l n}$ & $\delta_{\text {stat. }}$ & $\delta_{\text {uncorrel.syst. }}$ & $\delta_{\text {correl.syst. }}$ \\
\hline-2.33 & - & -2.00 & 0.0367 & 0.0057 & 0.0043 & 0.0047 \\
\hline-2.00 & - & -1.67 & 0.0216 & 0.0019 & 0.0024 & 0.0027 \\
\hline-1.67 & - & -1.33 & 0.0208 & 0.0016 & 0.0023 & 0.0026 \\
\hline-1.33 & - & -1.00 & 0.0133 & 0.0021 & 0.0015 & 0.0017 \\
\hline
\end{tabular}

and are close to zero for $x_{\gamma}^{\text {jet }}=1$. The hadronization corrections show only a weak dependence on $\eta_{\text {lab }}^{\text {jet }}$ and $x_{\pi}^{\text {jet }}$.

The LO and NLO calculations are shown with the measured cross sections in Fig. 7. After corrections for hadronization, there is good agreement between the NLO calculations and the measurements. The LO and NLO predictions without hadronization corrections do not describe the data.

\subsection{Ratios of leading neutron to inclusive dijet cross sections}

The ratio of the dijet cross sections with and without the requirement of a leading neutron, $f_{l n}$, is an interesting quantity in that it discriminates between the various Monte Carlo models used to describe leading neutron production. Further, if the hard interaction is independent of the neutron production, it should be essentially independent of the jet kinematics which reflect the hard process, neglecting possible phase space effects. As the only difference in the event selection for the leading neutron data and the inclusive dijet samples is the requirement of a leading neutron, some important systematic uncertainties cancel in the ratio. The remaining overall normalization uncertainties, mainly associated with the acceptance, efficiency and energy scale of the FNC calorimeter, are about $13 \%$.

For the photoproduction data, $f_{l n}$ is shown in Fig. 8 and in Table 4 as a function of the jet variables. Figure 8a shows that $f_{l n}$ is, within errors, independent of $E_{T}^{\text {jet }}$ and has an average value of about $2.3 \%$. However, as can be seen in Figs. $8 \mathrm{~b}-\mathrm{d}, f_{l n}$ shows a dependence on $\eta_{\text {lab }}^{\text {jet }}$, $x_{\gamma}^{\text {jet }}$ and $x_{p}^{\text {jet }}$. These dependences can only partly be reproduced by the PYTHIA model, which provides some estimate of the size of possible phase space effects. A better description of the ratio in Fig. 8 is possible, if the leading neutron data are described by the $\pi$-exchange model, RAPGAP- $\pi$, and the inclusive dijet data by PYTHIA-MI. This comparison suggests that the mechanism for dijet production in events with a leading neutron differs from that in inclusive dijet events.

This is further studied by measuring the $Q^{2}$ dependence of the ratio of the DIS dijet cross sections with and without the leading neutron requirement. The result is shown in Fig. 9 and in Table 5. Here, the point at $Q^{2}=0$ is the average of the ratios for photoproduction shown 

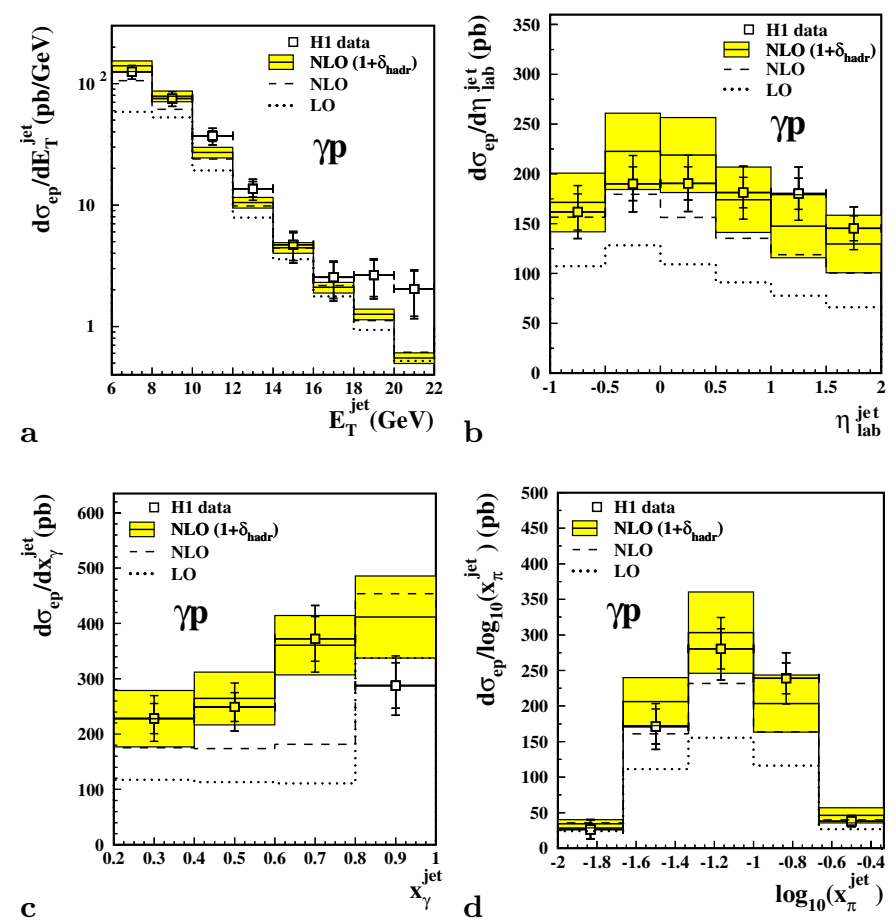

Fig. 7. The measured differential ep photoproduction cross section as a function of $E_{T}^{\text {jet }}, \eta_{\text {lab }}^{\text {jet }}, x_{\gamma}^{\text {jet }}$ and $x_{\pi}^{\text {jet }}$ for dijet events with a leading neutron. Inner error bars show the statistical errors, while the outer error bars represent the statistical and systematic errors, added in quadrature. The overall normalization uncertainty of $20 \%$ is not shown. LO, and NLO QCD predictions [10] before and after correction for hadronization effects, are compared with the measurements. The shaded bands show the quadratic sum of the factorization and renormalization scale uncertainties of the NLO predictions and of the uncertainty due to the hadronization corrections, $1+\delta_{h a d r}$. The kinematic regions within which this measurement is made are given in Table 1

in Fig. 8. Within the experimental errors, the RAPGAP model describes the measured ratio, when the leading neutron and the inclusive dijet data are represented by RAPGAP- $\pi$ and standard RAPGAP, respectively. However, there is some tendency for the measured ratio to increase with $Q^{2}$, for $Q^{2}$ below $20 \mathrm{GeV}^{2}$. A similar $Q^{2}$ dependence was observed by the ZEUS Collaboration in the analysis of inclusive DIS events with leading neutrons for not too large $x_{L}$ values [2].

\subsection{Discussion}

It was observed in [1] that pion exchange provides a good description of the semi-inclusive DIS process (1) in which a leading neutron is produced. The present results, given in Sects. 6.1 to 6.3, demonstrate that this is also the case for the small subsample of leading neutron events in which a dijet system is produced, in both DIS and photoproduction. This observation is not trivial, as the parameters used in the Monte Carlo models to empirically describe
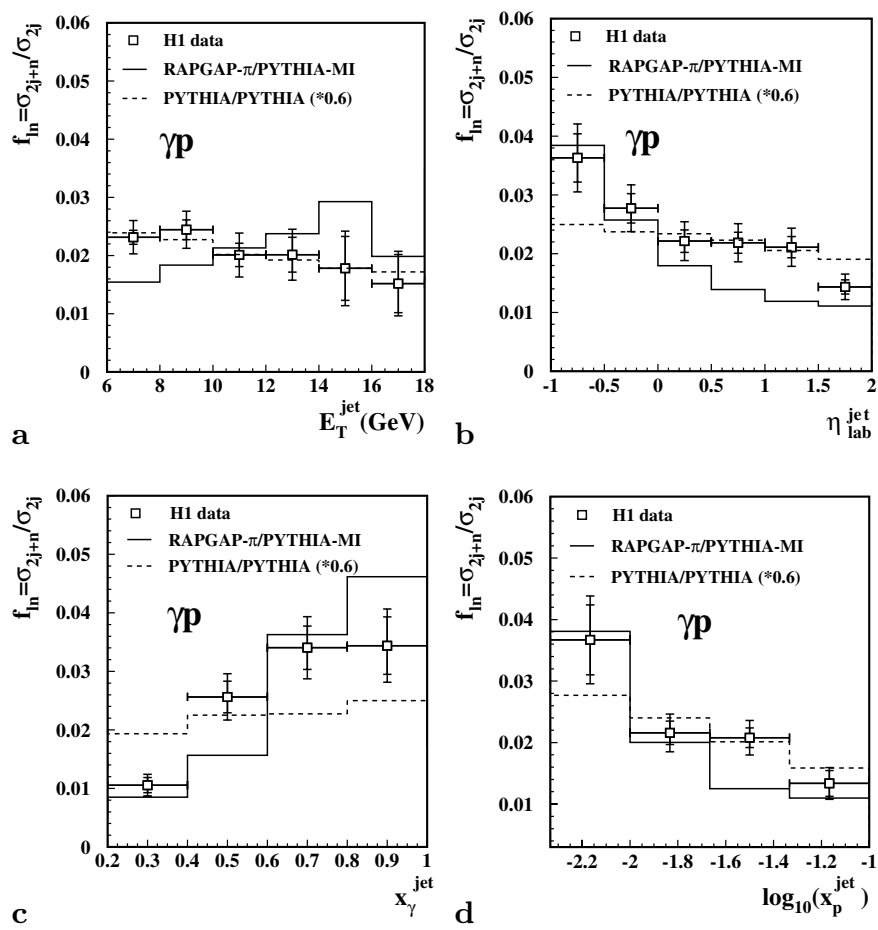

Fig. 8. The ratio of the cross section for dijet photoproduction with a leading neutron to that for inclusive dijet photoproduction, as a function of $E_{T}^{\mathrm{jet}}, \eta_{\mathrm{lab}}^{\mathrm{jet}}, x_{\gamma}^{\mathrm{jet}}$ and $x_{p}^{\mathrm{jet}}$. Inner error bars show the statistical errors, while the outer error bars represent the statistical and systematic errors, added in quadrature. The overall normalization uncertainty of $13 \%$ is not shown. Monte Carlo predictions for the ratios are obtained by using either RAPGAP- $\pi$ for the leading neutron cross sections and PYTHIA-MI for the inclusive cross sections, or by using PYTHIA in both cases. The PYTHIA prediction without multiple interactions has been scaled by a factor 0.6 to ease the shape comparison. The kinematic regions within which this measurement is made are given in Table 1

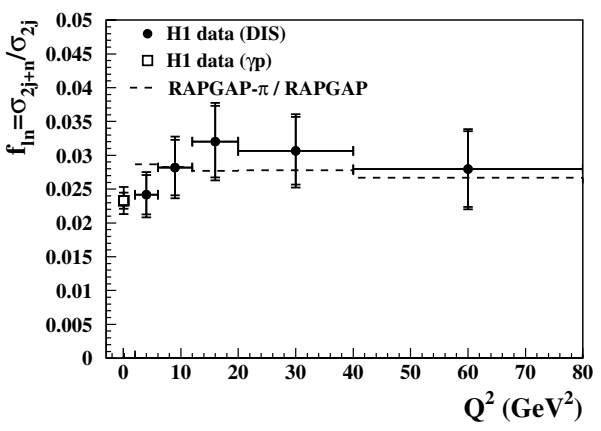

Fig. 9. The ratio of the cross section for dijet production with a leading neutron to that for inclusive dijet production, as a function of $Q^{2}$. Inner error bars show the statistical errors, while the outer error bars represent the statistical and systematic errors, added in quadrature. The overall normalization uncertainty of $13 \%$ is not shown. The Monte Carlo prediction, shown only for DIS, is obtained by using RAPGAP- $\pi$ for the leading neutron cross section and RAPGAP for the inclusive cross section. The kinematic regions within which this measurement is made are given in Table 1 
Table 5. The ratio of the cross section for dijet production with a leading neutron to that for inclusive dijet production, as a function of $Q^{2}$. The kinematic regions within which this measurement is made are given in Table 1

\begin{tabular}{ccccccc}
\hline \multicolumn{2}{c}{$\begin{array}{c}Q^{2} \\
{\left[\mathrm{GeV}^{2}\right]}\end{array}$} & $f_{l n}$ & $\delta_{\text {stat. }}$ & $\delta_{\text {uncorrel.syst. }}$ & $\delta_{\text {correl.syst. }}$ \\
\hline \multicolumn{2}{c}{$<$} & & & & \\
2 & - & 6 & 0.0241 & 0.0029 & 0.0017 & 0.0031 \\
6 & - & 12 & 0.0282 & 0.0041 & 0.0020 & 0.0061 \\
12 & - & 20 & 0.0320 & 0.0053 & 0.0022 & 0.0041 \\
20 & - & 40 & 0.0307 & 0.0050 & 0.0021 & 0.0039 \\
40 & - & 80 & 0.0279 & 0.0056 & 0.0020 & 0.0036 \\
\hline
\end{tabular}

the pion exchange were determined in hadronic reactions and no tuning to the present data was performed.

It is also observed that, for the DIS sample, the standard Monte Carlo models for the simulation of the hadronic final state, such as LEPTO and RAPGAP, predict cross sections for the production of dijets with a leading neutron which are too small. Again, no attempt to tune parameters was made. The increase of the leading neutron rate in dijet production caused by the introduction of non-perturbative soft colour interactions in LEPTO-SCI is not large enough to provide a good description of the measurements. However, LEPTO-SCI successfully describes the DIS reaction $e p \rightarrow e n X$ [1].

For the photoproduction sample, equally good descriptions are obtained with the pion exchange model RAPGAP- $\pi$ and the standard Monte Carlo program PYTHIA. The predictions of PYTHIA-MI clearly fail to describe the leading neutron data. However, the introduction of multiple interactions in PYTHIA is necessary to describe inclusive jet production [24]. The $x_{\gamma}^{\text {jet }}$ distributions in Fig. 5a demonstrate that this discrepancy between the data and PYTHIA-MI predictions is due to the poor description of resolved photon processes. The relative fraction of these processes is considerably lower in dijet production with a leading neutron than in inclusive dijet production. This finding is corroborated by the ratios of the dijet cross sections with and without the leading neutron requirement, presented in Fig. $8 \mathrm{c}$ as a function of $x_{\gamma}^{\text {jet. This }}$ ratio decreases by a factor of 3 as $x_{\gamma}^{\text {jet }}$ decreases from 0.9 to 0.3 , which could be explained by the increasing role of multiple interactions in inclusive dijet production at low values of $x_{\gamma}^{\text {jet}}$. The tendency seen in Fig. 9 for this ratio to rise with $Q^{2}$ may be due to absorptive corrections, as has been pointed out by several authors [34].

The differences between the dijet production data with and without leading neutrons, as well as the kinematic dependences of the cross section ratios, shown in Figs. 8b$\mathrm{d}$, point to differences in the production mechanism of events with and without leading neutrons. The present analysis shows that pion exchange is able to describe the properties of leading neutron events.

A similar analysis in the photoproduction regime has been published by the ZEUS Collaboration [11]. Qualita- tively, there is good agreement between the two studies. A detailed comparison is difficult, however, since the kinematic ranges and the jet algorithm used are different. The ZEUS Collaboration also observes that the ratio of leading neutron events to the inclusive sample is independent of $E_{T}^{\mathrm{jet}}$, and sees a similar dependence of this ratio on $x_{\gamma}^{\text {jet }}$. The dependence observed in [11] as a function of $\eta^{\text {jet }}$ is somewhat weaker than that in Fig. 8b.

\section{Summary}

The production of dijet events with a leading neutron of energy $E_{n}>500 \mathrm{GeV}$ and polar angle $\theta_{n}<0.8 \mathrm{mrad}$ is studied in photoproduction $\left(Q^{2}<10^{-2} \mathrm{GeV}^{2}\right.$ and $0.3<y<0.65)$ and in deep inelastic scattering $(2<$ $Q^{2}<80 \mathrm{GeV}^{2}$ and $\left.0.1<y<0.7\right)$. Dijet events with $E_{T}^{\text {jet1 }}>7 \mathrm{GeV}$ and $E_{T}^{\text {jet2 }}>6 \mathrm{GeV}$ are selected using a cone algorithm in the $\gamma^{*} p$ frame. The laboratory pseudorapidities of the jets are restricted to the region $-1<\eta_{\text {lab }}^{\text {jet } 1,2}<2$. Differential cross sections are presented as a function of $E_{T}^{\mathrm{jet}}, \eta_{\mathrm{lab}}^{\mathrm{jet}}, x_{\gamma}^{\mathrm{jet}}$ and $x_{\pi}^{\mathrm{jet}}$ for photoproduction, and as a function of $Q^{2}, E_{T}^{\mathrm{jet}}, \eta_{\mathrm{lab}}^{\mathrm{jet}}, x_{\gamma}^{\mathrm{jet}}$ and $x_{\pi}^{\mathrm{jet}}$ for DIS.

Both the cross section measurements and the neutron energy spectrum are reasonably well described by pion exchange models in which the photon interacts with a parton from the exchanged pion. These models are based on leading order QCD. The phenomenological parameters describing the pion exchange are taken from previous analyses of hadronic interactions. Next-to-leading order QCD calculations, after corrections for hadronization effects, also describe the measured photoproduction dijet distributions, in normalization as well as in shape. The experimental uncertainties are still too large to discriminate in this kinematic region between different parameterizations of the pion parton densities.

The standard LO Monte Carlo models which are not based on the pion exchange mechanism and which describe the inclusive jet production data, such as RAPGAP and LEPTO in DIS, and PYTHIA with multiple interactions in photoproduction, give a poor description of dijet production with leading neutrons. This remains true when soft colour interactions are added to LEPTO, although LEPTO-SCI is able to describe the inclusive leading neutron data. In photoproduction, there is no need to add multiple interactions, which are however necessary to describe the inclusive jet production data.

The ratios of the cross sections with and without the leading neutron requirement are studied as a function of $Q^{2}$ and of the jet kinematic variables listed above. If the hard process is independent of the leading neutron production, these ratios should not depend either on $Q^{2}$ or the jet variables. Indeed, there is no evidence for a strong dependence of the ratio on $Q^{2}$, or on $E_{T}^{\text {jet }}$ in photoproduction. However, the ratio in photoproduction rises with $x_{\gamma}^{\text {jet }}$. This suggests that the leading neutron dijet data have a lower fraction of resolved photon processes than do the inclusive dijet data. 
Acknowledgements. We are grateful to the HERA machine group whose outstanding efforts have made this experiment possible. We thank the engineers and technicians for their work in constructing and maintaining the $\mathrm{H} 1$ detector, our funding agencies for financial support, the DESY technical staff for continual assistance and the DESY directorate for support and for the hospitality which they extend to the non-DESY members of the collaboration. We wish to thank M. Klasen and G. Kramer for making their theoretical calculations available to us.

\section{References}

1. C. Adloff et al. [H1 Collaboration], Eur. Phys. J. C 6, 587 (1999) [hep-ex/9811013]

2. S. Chekanov et al. [ZEUS Collaboration], Nucl. Phys. B 637, 3 (2002) [hep-ex/0205076]

3. J.D. Sullivan, Phys. Rev. D 5, 1732 (1972)

4. H. Holtmann et al., Phys. Lett. B 338, 363 (1994)

5. B. Kopeliovich, B. Povh, I. Potashnikova, Z. Phys. C 73, 125 (1996) [hep-ph/9601291]

6. A. Szczurek, N.N. Nikolaev, J. Speth, Phys. Lett. B 428, 383 (1998) [hep-ph/9712261]

7. M. Przybycien, A. Szczurek, G. Ingelman, Z. Phys. C 74, 509 (1997) [hep-ph/9606294]

8. G. Ingelman, A. Edin, J. Rathsman, Comp. Phys. Commun. 101, 108 (1997) [hep-ph/9605286]

9. A. Edin, G. Ingelman, J. Rathsman, Phys. Lett. B 366, 371 (1996) [hep-ph/9508386]; A. Edin, G. Ingelman, J. Rathsman, Z. Phys. C 75, 57 (1997) [hepph/9605281]; J. Rathsman, Phys. Lett. B 452, 364 (1999) [hep-ph/9812423]

10. M. Klasen, G. Kramer, Phys. Lett. B 508, 259 (2001) [hep-ph/0103056]

11. J. Breitweg et al. [ZEUS Collaboration], Nucl. Phys. B 596, 3 (2001) [hep-ex/0010019]

12. T. Ahmed et al. [H1 Collaboration], Phys. Lett. B 348, 681 (1995) [hep-ex/9503005]

13. I. Abt et al. [H1 Collaboration], Nucl. Instrum. Methods A 386, 310 (1997); I. Abt et al. [H1 Collaboration], Nucl. Instrum. Methods A 386, 348 (1997)
14. B. Andrieu et al. [H1 Calorimeter Group], Nucl. Instrum. Methods A 336, 460 (1993)

15. R.D. Appuhn et al. [H1 SpaCal Group], Nucl. Instrum. Methods A 386, 397 (1997)

16. J.E. Huth et al., Towards a standardization of jet definition. Fermilab-Conf-90/249-E (1990)

17. T. Sjöstrand, Comp. Phys. Commun. 8274 (1994)

18. H. Jung, Comp. Phys. Commun. 86, 147 (1995)

19. P. Bruni, G. Ingelman, in: Proceedings of the Europhysics Conference, Marseille, France, July 1993, p. 595; (see also http://www3.tsl.uu.se/thep/pompyt)

20. M. Glück, E. Reya, A. Vogt, Phys. Rev. D 46, 1973 (1992)

21. M. Glück, E. Reya, A. Vogt, Z. Phys. C 53, 127 (1992)

22. C.F. Weizsäcker, Z. Phys. 88, 612 (1934); E.J. Williams, Phys. Rev. 45, 729 (1934); S. Frixione, M. Mangano, P. Nason, G. Ridolfi, Phys. Lett. B 319, 339 (1993) [hep$\mathrm{ph} / 9310350]$

23. T. Sjöstrand, M. Bengtsson, Comp. Phys. Commun. 43, 367 (1987); H.-U. Bengtsson, T. Sjöstrand, Comp. Phys. Commun. 46, 43 (1987); T. Sjöstrand, CERN-TH-6488 (1992)

24. S. Aid et al. [H1 Collaboration], Z. Phys. C 70, 17 (1996) [hep-ex/9511012]

25. G.A. Schuler, H. Spiesberger, in: W. Buchmüller, G. Ingelman (eds.) Proceedings of the Workshop Physics at HERA, Vol. 3 p. 1419 (1991)

26. A. Kwiatkowski, H. Spiesberger, H.J. Möhring, Comp. Phys. Commun. 69, 155 (1992)

27. G. Schuler, T. Sjöstrand, Z. Phys. C 68, 607 (1995) [hepph/9503384]; G. Schuler, T. Sjöstrand, Phys. Lett. B 376, 193 (1996) [hep-ph/9601282]

28. C. Adloff et al. [H1 Collaboration], Eur. Phys. J. C 13, 397 (2000) [hep-ex/9812024]

29. R.G.E. Timmermanns, Th.A. Rijken, J.J. de Swart, Phys. Rev. Lett. 67, 1074 (1991)

30. M. Glück, E. Reya, A. Vogt, Z. Phys. C 53, 651 (1992)

31. S. Aid et al. [H1 Collaboration], Z. Phys. C 69, 27 (1995) [hep-ph/9509001]

32. J.F. Owens, Phys. Rev. D 30, 943 (1984)

33. P.J. Sutton et al., Phys. Rev. D 45, 2349 (1992)

34. U. D'Alesio, H.J. Pirner, Eur. Phys. J. A 7, 109 (2000) [hep-ph/9806321]; N.N. Nikolaev, J. Speth, B.G. Zakharov, Preprint KFA-IKP(TH)-1997-17 [hep$\mathrm{ph} / 9708920]$ 